\title{
Retos comunicativos y educativos de las artes en los nuevos medios: el caso de la ópera
}

\section{Communication and educational challenges of the arts in new media: the case of the opera}

\author{
IVÁN LACASA \\ Facultad de Ciencias de la Comunicación. \\ Universitat Internacional de Catalunya. \\ lacasa@cir.uic.es \\ ISABEL VILLANUEVA-BENITO \\ Facultad de Ciencias de la Comunicación. \\ Universitat Internacional de Catalunya. \\ isabeluic@gmail.com
}

Recibido: 28 de marzo de 2012

Aprobado: 27 de junio de 2012

\section{Resumen}

En el siglo XXI, el deseo de las industrias artísticas es llegar a públicos más amplios y jóvenes. Movida por ese deseo e inmersa en la nueva era digital, la ópera ha decidido apoyar su estrategia de acercamiento a nuevas audiencias en la comunicación audiovisual y la educación. Este artículo analiza cómo está concretando esas dos líneas de actuación y se cuestiona si, al relacionarse con los medios, ya sea en su estrategia comunicativa, ya sea en su estrategia educativa, la ópera aprovecha toda la capacidad que estos tienen para conectar con los jóvenes y modular su cultura. Nuestro estudio concluye que, para alcanzar los objetivos que se ha marcado, la ópera debe reorientar su estrategia, dar un paso adelante y afrontar una auténtica audiovisualización mediática de sus acciones, en lugar de seguir centrándose en explotar por más canales la capacidad que los medios tienen de transportar el espectáculo en vivo. Ese es ahora su auténtico reto comunicativo y educativo.

Palabras clave: òpera, medios, públicos, audiovisual, educación, artes.

Lacasa, I. y Villanueva-Benito, I. (2013): Retos comunicativos y educativos de las artes en los nuevos medios: el caso de la ópera. Arte, Individuo y Sociedad, 25(2) 219-232

\begin{abstract}
In the XXI century, art industries aim to reach younger and wider audiences. Driven by this aspiration and immersed in the new digital era, opera has decided to base its strategy to approach larger audiences on audiovisual communication and education. This article discusses how these two lines of action are taking shape and questions if in its relationships with the media, either in its communicative or educational schemes, opera sufficiently knows how to take profit from all the abilities of the young to connect with them and modulate their culture. Our study concludes that in order to achieve the objectives it has set for itself, opera should refocus its strategy, step up and face a real mediatic audiovisualisation of its actions, rather than trying to exploit through more channels media's capacity to broadcast on live. In short, this is nowadays opera's real communicative and educational challenge.
\end{abstract}

Key Words: ópera, media, audiences, audiovisual, education, arts.

Lacasa, I. y Villanueva-Benito, I. (2013): Communication and educational challenges of the arts in new media: the case of the opera. Arte, Individuo y Sociedad, 25(2) 219-232 
Sumario: 1. Introducción: la era digital hace posible una educación cultural mediática, 2. La estrategia comunicativa de la ópera, 3. La estrategia educativa de la ópera, 4. Conclusión: el reto de la audiovisualización mediática. Referencias.

\section{Introducción: la era digital hace posible una educación cultural mediática}

En los últimos años, los investigadores y analistas han acudido a conceptos como «globalización», «mercantilización», «convergencia», «digitalización»o «audiovisualización» para tratar de explicar los profundos e imbricados procesos de cambio que se están dando en la economía, la política, la cultura o la sociedad (Hasebrink y otros, 2004, p. 9; Miège, 2006; Bustamante, 2011, p. 61; Bakhshi y Trosby, 2010, p. 13-16). La realidad hipermediada y pantallizada en la que viven los públicos (Lipovetsky y Serroy, 2009; Fernández Quijada, 2007) modifica sus hábitos y provoca que, tanto el aprendizaje del mundo en el que viven como las acciones que sobre él ejercen, queden fuertemente determinados por el uso, cada vez más activo, de los medios de comunicación audiovisual (Lundby, 2009, pp. 1-15; Fontcuberta, 2003; Martín Barbero, 2002).

En un intento de aprovechar el entorno y de readaptar sus formas productivas a los nuevos públicos, medios y mercados (Cardoso 2009, p. 157), las industrias culturales, también las europeas, están abriendo su discurso al nuevo consumidor, que es mediático, activo y sobre todo audiovisual (Krotz 2007; Hicketier 2010). El sector de la ópera, consciente además de que tradicionalmente se le ha asociado a la alta cultura (Adorno, 2006; Tambling, 1987), ha visto doblemente necesario no permanecer ajeno a esa evolución y ha iniciado un complejo proceso dirigido a remover la imagen de distante que los grandes públicos y, en especial los jóvenes, han construido de ella (Radigales, 2005, p. 59; Roselli, 1998, pp. 450-451). Según puede leerse en sus sitios oficiales, los principales teatros europeos manifiestan un deseo expreso de diversificar los perfiles de sus consumidores y, sobre todo, de acercarse a más amplias y jóvenes audiencias (pueden consultarse, entre otros, www.eno.org; www.metoperafamily.org; www.operaxxi.com/protagonistas). A fin de lograr tal cometido, la industria de la ópera innova poniendo en práctica, de manera más o menos coordinada, dos grandes líneas de acción: difundir el arte a través de nuevas estrategias comunicativas y educar en su obra a las nuevas generaciones.

\section{La estrategia comunicativa de la ópera}

Las iniciativas comunicativas, publicitarias y promocionales propias de la aplicación del márquetin a las artes escénicas están ayudando a las industrias culturales a aumentar ingresos, a reducir gastos y, sobre todo, a ampliar audiencias (Yorke \& Jones, 1984; Cuadrado, 2001; McLean, 1994; Scheff \& Kotler, 1996). A la vista de la utilidad de los principios que esas disciplinas enseñan (Colbert \& Cuadrado, 2003), también la ópera quiere hacer un uso intensivo de los medios de comunicación para conquistar nuevos mercados y expandir sus horizontes más allá de los teatros (Heyer, 2008, pp. 601-602). Le ayudan en ello sus relaciones naturales con el arte audiovisual. En efecto, numerosos autores han descrito a la ópera y al discurso audiovisual como dos artes de 
artes basadas en un conjunto de disciplinas coordinadas en armonía para transmitir una síntesis narrativa superior. Gracias a diferentes oficios artísticos puestos en escena para conseguir recrear el anhelo de un universo narrativo, tanto en la ópera como en el discurso audiovisual entran en juego sonidos, música, imagen, color y movimiento (Ishaghpour 1995, pp. 27-31; Radigales, 2005; Adorno, 2006). En consecuencia, la ópera comparte con la ficción, ya sea en el cine, en la televisión, el vídeo o internet, el rasgo de estar basadas en una temporalidad narrativa (Citron, 2000; Fawkes, 2000; Schroeder, 2002). Justo esa esencial dimensión narrativa y ficcionada de la ópera parece corresponderse plenamente con el tipo de consumo que demuestra tener el público joven al que ella ahora quiere llegar (Medrano Samaniego \& Cortés Pascual, 2007). Así, la ópera, igual que otras industrias culturales, ha decidido apostar por la comunicación audiovisual y explora nuevas estrategias de negocio distribuyendo su producto a través de múltiples ventanas de difusión (Berini, 2011; Radigales, 2005, pp. 59-61; Radigales \& Villanueva, 2011). El espectáculo original ofrecido por los teatros en directo y en comunidad, considerado el principal motor artístico del género musical, convive ahora con una nueva oferta mediática operística (Parker, 1994, p. xi, Roselli, 1998, p. 482). Asistir al directo ya no constituye la única de las opciones de consumo ni, por tanto, la única forma de conseguir la «participación afectiva» de los públicos en el espectáculo operístico (Gordillo, 2008, pp. 9, 10).

Sin embargo, la industria de la ópera no ignora que la atención que concentran las tecnologías y los medios audiovisuales de los que ha decidido servirse podría disminuir la asistencia al espectáculo en vivo y debilitar, con ello, su motor artístico (Föhl \& Lutz 2010, p. 28). Por ende, cuando se relaciona con los medios, procura mantenerse lo más objetiva posible y trata de que el uso de las técnicas audiovisuales no interfiera en la grabación, a fin de respetar las experiencias vivibles en el directo y de satisfacer, con ello, los gustos del espectador ya operófilo (Senici, 2010; Heyer, 2008). Como consecuencia, los productos audiovisuales que la ópera ofrece, derivados de la grabación en el teatro, parecen quedar algo condicionados, técnica y artísticamente, por la primacía de la (re)transmisión del espectáculo original (Morris, 2010, pp. 98-99; Senici, 2010, pp. 63-80). Aunque ha previsto que asistir al directo deje de ser la única opción para disfrutar de su arte, la relación que de hecho ha establecido la ópera con los medios de comunicación es asimétrica, pues los llama a servir y a evocar a su motor artístico, nunca a competir con él. Con ello parece distinguir en su oferta, al menos implícitamente, una jerarquía compuesta por productos audiovisuales que, si bien es variada, en el fondo podría ser descrita como el despliegue de un único producto operístico originario presencial dado a conocer gracias a una multiplicación de ventanas de difusión. Del irreemplazable producto primario (ópera en vivo y en comunidad) surgen entonces los derivados mediáticos, a los que llamaremos productos operísticos secundarios y terciarios (Lacasa \& Villanueva, 2011, pp. 65-74).

Por un lado, los productos operísticos secundarios serían las óperas grabadas, distribuidas y exhibidas a través de las tecnologías y los dispositivos propios de la comunicación audiovisual, así como materializadas en cualquiera de sus formatos de consumo. Hoy satisfacen consolidados nichos de mercado. Un caso muy destacado es el negocio emergente de la ópera en los cines que el Metropolitan Opera House 
de Nueva York ha conseguido extender a más de 46 países del mundo. Aunque el proyecto fue presentado en 2008 como aquel capaz de hacer «accesible la ópera a todas las capas sociales», hasta el momento no puede decirse que haya favorecido la generalización del arte, pero sí su descentralización, puesto que ya no es necesario desplazarse hasta un teatro para disfrutar de la ópera en directo y basta con acudir a los cines más cercanos que ofrezcan el servicio (Sánchez, 2008, p. 36; Herrscher, 2009, pp. 24-25).

La industria del DVD y la de los canales de televisión de pago, cada vez más permeables a la búsqueda de fórmulas audiovisuales de fidelización de los nuevos públicos mediáticos, constituyen un segundo caso digno de mención. Citamos, como ejemplo, dos de las adaptaciones incorporadas en estos negocios: la división de la obra en unidades más discretas de información (para la fácil navegación y selección de escenas) y la incorporación de extras, aquellos elementos que ayudan a entender la ópera como un arte mucho más enriquecedor y complejo que lo que puede apreciarse en el hecho musical mismo: entrevistas a los creadores, documentación relativa a la obra y, sobre todo, making of (De Diego, 2010, pp. 8-9; Radigales \& Villanueva, 2011). Todas ellas son herramientas destinadas a satisfacer el deseo de consumir una información que aporte valor añadido, a educar y a provocar una fidelización indirecta en el consumidor, así como, naturalmente, a favorecer la compra del producto.

La ópera audiovisual disponible en la Red a través de dispositivos de video on demand o de ópera en directo in streaming, merecen también un comentario. Aunque Internet se presente a priori como un medio poco adecuado para consumir productos operísticos secundarios, es decir, óperas audiovisualizadas de larga duración, las retransmisiones in streaming conservan algo de la dimensión vivencial del evento en directo y en comunidad (Ramírez, 2011). Esta última característica permite la exploración de nuevos negocios, tales como el Palco Digital, estrenado el pasado junio por el Teatro Real de Madrid, que posibilita al usuario disfrutar de los estrenos del teatro desde su ordenador doméstico. Por otro lado, el consumo de vídeo a la carta, cada vez más personalizado y sofisticado, ha llevado a diseñar auténticas mediatecas artísticas. El Metplayer, ofrecido por el Metropolitan Opera House de Nueva York, reúne una colección de más de 300 producciones audiovisuales en HD, y ofrece además recuperaciones de vídeo analógico y grabaciones sonoras, lo cual confiere al proyecto un elevado valor documental.

Por su parte, los productos terciarios serían todas aquellas manifestaciones audiovisuales que, en lugar de presentar la obra artística en su totalidad, ofrecen partes fragmentadas o modificaciones de la misma. En esta tercera tipología incluimos también todos los productos on-line cuyo contenido expreso no es ópera pero sí está relacionado directamente con ella. Sin que puedan ser considerados propiamente ópera, los productos terciarios constituyen una herramienta de comunicación necesaria para publicitar el género y ampliar la presencia de este arte. Lo hacen no solo formando parte de una estrategia de márquetin, sino confiriéndole un valor cultural en tanto que mundo de referencia al que las personas pueden asociar activamente sus vidas, relaciones, fantasías y deseos, los cuales, entonces, pasan a fluir por los canales de los medios (Jenkins, 2008, p. 27). De los productos terciarios que tienden a estandarizarse en el mercado, han cobrado fuerza representativa el making of 
on-line de las obras, los vídeos sobre la vida de las estrellas líricas que alimentan el Star System operístico, o la incorporación de prácticas y formatos televisivos en las retransmisiones, tales como el reality show, los trailers promocionales, los videoclips líricos, o el uso de podcasts, entre otros (De Diego, 2010, pp. 1-2; Heyer, 2008, p. 596).

Todas estas manifestaciones mediáticas secundarias y terciarias certifican que son muchas las oportunidades que se presentan para trazar caminos entre la ópera y los grandes públicos audiovisuales. Cabe preguntarse por qué, a pesar de esa diversificación de ventanas de difusión, el consumo mediático de la ópera sigue en gran parte limitado al sector de los públicos ya fidelizados: operófilos con inquietudes coleccionistas en el caso del consumo del DVD, amantes del género en el caso de los canales de pago en televisión, asistentes asiduos al directo en el caso de la ópera en los cines e Internet, por ejemplo (Heyer, 2008, pp. 600-603; De Diego, 2010; Morris, 2010, pp. 96-99; Berini, 2011). La todavía tímida respuesta de las nuevas audiencias a las iniciativas comunicativas que acabamos de exponer quizás no se deba solo a una falta de educación en la ópera, sino también a que esta sigue sin ofrecer una verdadera diversificación de contenidos audiovisuales que responda con coherencia a las necesidades de lectura de los nuevos públicos, a pesar de la palpable multiplicación de plataformas de difusión que ha emprendido. Puede, en efecto, que el problema radique en que está confundiendo audiovisualizarse mediáticamente con mediatizar el vivo o, dicho de otra manera, en que está centrando «en el medio audiovisual y no en el lenguaje audiovisual» la comunicación que quiere establecer con los públicos jóvenes (Bartolomé, 1987, p. 51). Es un hecho que la tendencia general en todos estos productos secundarios y terciarios es ofrecer una grabación tipo documento de la obra, en la cual el lenguaje audiovisual intenta mantenerse objetivo para no modificar la esencia de la producción en directo y emular las experiencias vividas en el teatro (D'Agostino, 2011; Morris, 2010). De la misma manera, elementos como el montaje audiovisual y su ritmo, la escenografía e iluminación, los ángulos y tamaños de los planos o puntos de vista más subjetivos se siguen subordinando a la recreación del vivo, en un intento de no perder la esencia del original (Senici, 2010; Bové, 2011). Con ello, la ópera reduce la expresión fílmica de sus obras a la mera difusión y olvida, en gran parte, que todo mensaje mediático pleno se confecciona cumpliendo convenciones formales y códigos típicos de la narrativa audiovisual (Romea, 2001, pp. 71-78). Por el contrario, habiendo sido alfabetizados nativamente en el audiovisual, los públicos a los que ahora se dirigen las industrias culturales entienden e interpretan los contenidos de los medios a partir de códigos y aspectos formales distintos a aquellos que la cultura y las artes experienciadas en su espacio original de representación ponen en juego (Pereira Domínguez \& Urpí Guercia, 2005, p. 77). Un cuadro, una música, el decorado, la escenografía y, en definitiva, cualquier elemento que aparece a través de la pantalla «se halla comprometido narrativamente con los hechos filmados y adquiere, por ese motivo, un significado específico en la comunicación entre la película y el espectador» (Mitry, 1978, pp. 164-165). Si quieren tener éxito en su proceso de adaptación a los nuevos medios, las artes deben ser conscientes no solo de sus propias intenciones, condiciones y posibilidades técnicas, sino también - y sobre todo- de los rasgos y expectativas de los públicos 
que los usan. La respuesta de estos últimos es la que recordará a la ópera que no está en su mano consolidar la jerarquía del producto primario mediante la estrategia a la que hasta ahora ha acudido: introducir una disociación entre los contenidos de la obra en vivo que mediatiza y el componente audiovisual formal, o, lo que es lo mismo, tratar de convertir a este último en algo meramente descriptivo. Más aun: si quiere conectar con los públicos jóvenes, la ópera debería favorecer, en primer lugar, que estos pudieran apreciar su contenido - fruto de un arte que les es desconocido y complejo- a través de una forma más audiovisual, esto es, más familiar para ellos.

En definitiva: sin necesidad de renunciar a las grabaciones derivadas del directo, la industria operística podría aprender de los lenguajes propios de los medios y ampliar su oferta con productos secundarios y terciarios que fueran formalmente audiovisuales y, por tanto, identificables y valorables como realmente mediáticos y familiares para los nuevos consumidores. En realidad, construir discursos operísticos menos teatrales y más propios del lenguaje mediático audiovisual e ir más allá de una filmación tipo documento, antes que significar una renuncia a la actual oferta de espectáculo mediado (la ópera en los cines, por ejemplo), puede implicar su ampliación, para dar así satisfacción a nuevos nichos de interés mediante experiencias artísticas que serán vistas como novedosas, por estar basadas en la ópera como un espectáculo audiovisual (Senici, 2010; Esse, 2010; Morris, 2010). Un motivo adicional refuerza la importancia de esa ampliación de la oferta operística actual: gracias a la proliferación de soportes digitales, es el producto resultante de la mediación audiovisual del directo - es decir, el producto operístico secundario o terciario- el que será capaz de perdurar en el tiempo y de quedar a disposición de los públicos mediáticos de forma indefinida. Todo lleva a pensar, en conclusión, que el reto comunicativo que hoy afronta la ópera es armonizar mejor sus productos secundarios y terciarios con los usos que los públicos hacen de los medios y de sus contenidos.

\section{La estrategia educativa de la ópera}

La estrategia comunicativa recién descrita cumple ya una cierta función educativa, pues a través de los medios la ópera se da a conocer. Gracias a una imagen más moderna y una presencia mediática más constante y algo más sintonizada con las nuevas generaciones, el arte operístico está alejando de sí ciertos prejuicios sociales referidos a su dimensión económica, estética e incluso política, que ha heredado de épocas anteriores (Adorno, 2006; Mordden, 1985; Roselli, 1998; Laenen, 2003, p. 11). Pero esta necesidad educativa que la ópera experimenta posee un origen mucho más trascendente que el deseo de mejorar su imagen. Cuando la ópera educa a los públicos está capacitándolos para que puedan ejercer más plenamente el derecho humano fundamental de acceso a la cultura (Gaudio, 2009, pp. 57-65). No en vano, cuando la ópera forma, lo hace en ella misma y en otras muchas artes. El aprendizaje de la ópera, arte de artes, facilita en los públicos apreciaciones estéticas, emotivas y narrativas aplicables también a la música, la danza, el teatro, el cine, las artes visuales y a todas aquellas disciplinas integradas en ella (Smith, 1993, p. 21; Roselli, 1998, pp. 450-482; Alier, 2008).

Son muchas las iniciativas que están llevando a cabo los organismos culturales para educar en el género musical a las nuevas generaciones. Citamos por su valor 
ilustrativo las acciones que el Metropolitan Opera House y la red Opera America ha realizado en cientos de colegios de Estados Unidos. En Europa deben destacarse, a su vez, proyectos de colaboración entre teatros y organismos educativos, así como iniciativas de educación infantil a través de la Red. Apuntamos las iniciativas del Petit Liceu y el Petit Palau en las escuelas de Barcelona. Asimismo destacamos el proyecto digital Inside Out de la English National Opera, los proyectos infantiles de la Scottish Opera, y la web lanzada por la Welsh National Opera para dar a conocer el arte a los jóvenes (pueden consultarse, como ejemplo, los sitios oficiales de los casos citados: www.scottishopera.org; www.liceubarcelona.cat; www.metguild.org).

También es obligado referir aquí el intento colectivo de unificar las iniciativas educativas en Europa que, en 1996, dio lugar a la Red Europea de Educación de Ópera y Danza (RESEO), a la que hasta hoy se han adscrito más de 70 teatros de referencia internacional. Desde el comienzo de su andadura, RESEO constituyó la principal plataforma de intercambio de buenas prácticas en la educación del arte operístico en más de 20 países. RESEO coordina, promueve y evalúa científicamente, además, proyectos de educación operística infantil y juvenil. Con sus actividades favorece, por añadidura, la construcción de un sentimiento europeo conjunto que revalorice sus artes más propias e identitarias (Martel 2011, pp. 415-443). Debemos mencionar, en primer lugar, las óperas participativas y los proyectos de estimulación musical para preescolares. Aunque las instituciones adscritas a RESEO que ofrecen programaciones para niños menores de 3 años todavía son solo tres (TRAFFO CarréRotondes, Gran Teatre del Liceu y la Opéra National de Bordeaux), este tipo de iniciativas comienza a emerger con fuerza (Gaudio, 2009, p. 41). En segundo lugar, destacamos las óperas programadas especialmente para niños que RESEO impulsa. Constituyen proyectos multidisciplinares en los que se estudia la viabilidad de una ópera destinada a todos los públicos, se promueven descuentos en los precios y se fomenta la participación de los niños en el proceso creativo de la producción, entre otras actividades (Gaudio, 2009, pp. 7-68). Aludimos, también, a las colaboraciones experimentales internacionales, en las que varios teatros y colegios colaboran en producciones temáticas. En 2003 fue el caso de Why/How education to opera today in Europe, en 2005 el de Creative Ways to Mozart y en 2012 será el de Intergenerational Work. En el ámbito universitario, por su lado, los proyectos de RESEO promueven tres acciones principales: conocer el teatro y a los artistas, ver o asistir a las representaciones, y participar en los ensayos. Citamos, a modo de ejemplo, los proyectos participativos con estudiantes, la creación de paquetes económicos que ofrecen la entrada y la comida en los teatros, las visitas a ensayos de orquesta, así como la creación de debates y workshops de ópera con los artistas (Nöel, 2010).

El análisis de la oferta educativa de los teatros adscritos a RESEO confirma que lo que de hecho hacen es promover una educación en el producto operístico primario, sin tener demasiado en cuenta los productos operísticos audiovisuales - aquellos a los que en el apartado anterior hemos llamado secundarios y terciarios. En casi todas las iniciativas que hemos analizado, cuando los teatros usan los medios de comunicación, lo hacen atendiendo, casi exclusivamente, a su faceta de instrumentos de difusión del espectáculo en vivo (O’Shaughnessy, 2006). La explicación de este hecho parece radicar en que, a pesar de la voluntad unificadora de RESEO, la cultura 
finalmente «no tiene una configuración unitaria», sino que «se divide y diferencia tantas veces como lo hacen los grupos humanos» (Solé Blanch, 2005, p. 482), por lo que el arte universal de la ópera tiende a materializarse y vivirse a través de las industrias culturales de cada territorio. Resulta lógico, por tanto, que sean los teatros, en tanto que instituciones que programan las temporadas de ópera, quienes también promuevan la educación en el arte y le den un sentido beneficiario para su propia comunidad (Ramírez, 2011). Este hecho se traduce en que, al menos en un primer momento, las iniciativas educativas de cada comunidad van destinadas a la promoción de la asistencia a las salas y, por tanto, a la valoración del espectáculo en directo, puesto que el vivo constituye el motor económico y artístico de esos teatros.

El poco uso que de las tecnologías digitales suelen hacer en sus acciones educativas responde, en definitiva, al planteamiento de fondo que, tal como hemos visto, ya caracterizaba a la estrategia comunicativa de la ópera: la priorización del vivo. Sin embargo, si contrastamos dicha priorización con la condición de los destinatarios de esos programas educativos, la manera de proceder de dichos teatros encierra una contradicción: pretenden dirigirse a los jóvenes, pero lo hacen arrinconando los elementos mediáticos, utilizando solo con cierta frecuencia las consultas de páginas web, la ilustración mediante el uso del DVD en clase, o los juegos virtuales multimedia (O'Shaughnessy, 2006). De ahí que sean tan relevantes casos internacionales como el de Opera Oberta en Cataluña, un proyecto colaborativo entre las universidades, los teatros y el I2CAT, que hace un uso intensivo de los medios audiovisuales. El proyecto utiliza los productos secundarios de la ópera para divulgar el género entre los estudiantes; explica el arte mediante la retransmisión del espectáculo y, posteriormente, profundiza en el seguimiento del aprendizaje musical mediante la utilización de herramientas multimedia a través de los nuevos medios. De acuerdo con Enrique Fuentes (2010), su éxito se debe a que «integra la ópera en ciertos rituales de la vida profesional y personal de los jóvenes», tales como el consumo de los medios, «sintonizándose con la forma en que hoy en día adquieren los conocimientos» (Fuentes, 2010, pp. 44-45).

El éxito de Opera Oberta indica, por contraste, que, al menos hasta el momento, en otros muchos proyectos la ópera está perdiendo la oportunidad de explotar el potencial educativo de los medios de comunicación (Pérez Tornero, 1994; Marcos Ramos, 2010; Fontcuberta, 2005, p. 154). Ciertamente, las estrategias basadas en el directo resultan muy motivadoras para los asistentes, pero, insertas como están en una sociedad mediática, si se apoyan de forma casi exclusiva en la presencialidad del vivo, poseen un alcance limitado, porque resultan difíciles de integrar en la vida y mundos cognitivos y afectivos de los posibles nuevos públicos a los que quiere llegar (Silverstone 1994, p. 3; García Avilés, 2009). Las industrias artísticas han de entender que el proceso de percepción mediático-audiovisual, al que nos hemos referido en el apartado anterior, comienza siempre a través de los estímulos formales que la obra ofrece (Pereira Domínguez \& Urpí Guercia, 2005, p. 78). Al haberse acostumbrado a consumir expresiones fílmicas, los jóvenes buscarán intenciones comunicativas que añadan significación al lenguaje audiovisual utilizado en los productos operísticos mediatizados que hoy comienzan a extenderse (Mitry, 1978, p. 330). Si como receptores no pueden realizar una buena lectura audiovisual, por no ser 
esos productos suficientemente audiovisuales, tampoco lograrán captar la intención y propiedades del mensaje (Aparici, 1995, pp. 7-15). Y solo si son capaces de otorgar significación a la forma de los productos operísticos, los públicos, que hoy son más mediáticos que operísticos, acabarán experimentando gratificaciones de especies superiores con ese arte, a saber, estéticas, emotivas o narrativas, entre otras (Ferrés, 1997). En caso de que en sus programas educativos la ópera facilite una lectura formal de su espectáculo audiovisualizado, esto es, en caso de que surja una oferta de productos operísticos secundarios y terciarios que se alejen del vivo, se adapten realmente a las formas de lectura mediatico-audiovisual y ganen presencia también en los programas educativos de la ópera, dichos productos lograrán constituir nuevas formas de organizar emociones populares, más ligadas a los lenguajes y significados mediáticos que al espectáculo original (Gordillo, 2008, pp. 9-10). El beneficio social será patente: dado que la ópera es un arte basado en el «placer de sentir las emociones estéticas» (Rössel, 2011, p. 89), su impacto educativo será no solo formal, sino también emocional, lo que hará que los vínculos que el espectador establezca con el mundo del arte no consistan en «frías abstracciones mentales, sino en auténticas implicaciones personales» (Pereira Domínguez \& Urpí Guercia, 2005, p. 78).

La familiarización con la ópera, no importa si ha sido alcanzada a través de una enseñanza más audiovisualizada, conllevará una modificación positiva de la imagen social de este arte (Yorke y Jones, 1984; Cuadrado, 2001, pp. 80-81) y, por añadidura, la transformación de la asistencia a los teatros en un acontecimiento mucho más natural en la vida de los jóvenes. Gracias a la fuerza intrínseca del vivo, tras la identificación de los públicos jóvenes con el arte a través de la oferta audiovisual, el motor artístico de la ópera quedará revalorizado: los nuevos públicos, por fin más predispuestos y preparados para apreciar el arte, en ningún caso descartarán asistir al directo (Benhamou, 2009, p. 14). El hecho de que la ópera ofrezca unos productos y a unos programas educativos más en sintonía con sus hábitos, lenguajes y sensibilidades, multiplicará la posibilidad de que pasen a contemplar el vivo de manera aspiracional y a querer vivirlo como «un momento de convivencia y de emociones compartidas» (Lipovetsky \& Serroy, 2009, p. 65).

En definitiva: la audiovisualización mediática se presenta como un paso imprescindible para que los públicos jóvenes se identifiquen más fácilmente con formas artísticas cercanas a sus hábitos y expectativas, sepan construir significados a partir de ellas y obtengan unas gratificaciones que, al ser superiores y capaces de provocar un impacto emocional, les vinculen personal y afectivamente al arte. La familiarización de los grandes públicos con la ópera a través de los productos secundarios y terciarios, ya sea a nivel comunicativo, ya sea a nivel educativo, añadirá valor aspiracional a la cultura, además de al espectáculo primario en directo y en comunidad.

\section{Conclusión: el reto de la audiovisualización mediática}

Tras analizar la manera en que la ópera está concretando sus líneas de actuación comunicativa y educativa, concluimos que aquello que más aprecia de los medios es su capacidad de transportar el vivo, no sus valores audiovisuales mediáticos. Por ello, ni 
en la estrategia comunicativa, ni en la educativa, aprovecha a fondo el gran potencial modelador de cultura que en nuestros días posee el componente audiovisual.

Hoy el reto comunicativo y educativo de la ópera es un reto audiovisual. Si la ópera se audiovisualizara mediante productos secundarios y terciarios, su oferta se diversificaría y completaría, se tornaría más personalizada y social. La presencia en el mercado de productos operísticos auténticamente mediáticos que, sin dejar de ser ópera, ya no estuvieran directamente condicionados por el vivo y tuvieran identidad audiovisual propia, ayudaría a generar un mundo de referencia con el que los públicos mediáticos podrían identificarse más fácilmente. La presencia en los programas formativos de productos operísticos secundarios y terciarios, que complementaran el protagonismo didáctico otorgado al directo, serviría para hablar mediante un idioma común y familiar a esos públicos alfabetizados en los lenguajes audiovisuales. La audiovisualización mediática está llamada a jugar un papel no solo en la calidad de la apreciación comunicativa de la ópera, sino también en su trascendental misión educativa y fidelizadora: una identificación y familiarización que partiera de la adaptación - que no mutación - formal, permitiría al público apreciar el producto operístico concreto e identificarse globalmente con la ópera en tanto que gran arte (Roselli, 1998, p. 482; Tomlinson, 2001, p. 10).

No queremos cerrar estas líneas sin subrayar que el reto audiovisual al que la ópera se enfrenta es sobre todo una gran oportunidad social. Significaría, en efecto, un claro bien colectivo que el arte multidisciplinar de la ópera diera un paso adelante y afrontara el desafío de adaptar con más profundidad su actividad a las formas mediático-audiovisuales en que los nuevos públicos consumen y aprenden. Como la ópera es un arte que combina e integra a otras muchas artes, la audiovisualización resaltaría su poder para «expresar, intensificar, e iluminar los sentimientos y experiencias de la raza humana» de una manera más profunda que la que ninguna de ellas puede conseguir por separado (Smith, 1993, p. 21). El aprendizaje audiovisualizado del que es considerado un arte de artes facilitaría en los nuevos públicos apreciaciones estéticas, emotivas y narrativas que, además, quedarían asociadas a los medios, algo que, sin duda, los dignificaría. La familiarización con una ópera audiovisualizada ayudaría a conseguir una alfabetización mediática más llena de contenido. Generaría audiencias que demandarían a la sociedad y a los medios poder acceder a contenidos de un nivel cultural más elevado. En los teatros y en el día a día.

\section{Referencias}

Adorno, T. (2006). Escritos musicales I-III. Madrid: Ediciones Akal.

Alier, R. (2008). ¿Qué es esto de la Ópera?. Barcelona: Ediciones Robinbook.

Aparici, R. (1995). Educación Audiovisual. La enseñanza de los medios de la escuela. Buenos Aires: Ediciones Novedades Educativas.

Bakhshi, H. \& Trosby, D. (2010). Culture of innovation. An economic analysis of innovation in arts and cultural organisations. London: NESTA.

Bartolomé, A. (1987). Análisis de la producción y aplicación de programas audiovisuales didácticos. Barcelona: Universidad de Barcelona. 
Benhamou, F. (2009). Economía y Cultura: nuevas perspectivas. En C. Asuaga (Ed.), Cultura, Ciencias Económicas y Derecho: un encuentro no casual (pp.1120). Montevideo: Fundación de Cultura Universitaria.

Berini, B. (2011). Entrevista realizada por los autores al departamento audiovisual y de documentación del Gran Teatre del Liceu. Barcelona.

Bové, X. (2011). Entrevista realizada por los autores al departamento audiovisual y de documentación del Gran Teatre del Liceu. Barcelona.

Bustamante, E. (2011). Cultura digital: la "nueva" cultura clásica. Telos, 88. Recuperado de http://sociedadinformacion.fundacion.telefonica.com/ seccion $=1268 \&$ idioma $=$ es ES\&id $=2011072709090001 \&$ activo $=6$.do

Cardoso, G. (2009). Los medios de comunicación en la sociedad en red. Filtros, escaparates y noticias. Barcelona: UOC.

Citron, M. (2000). Opera on Screen. London: Yale University Press.

Colbert, F. \& Cuadrado, M (Eds.). (2003). Marketing de las Artes y la Cultura. Barcelona: Ariel.

Cuadrado, M. (2001). La gestión de marketing en las entidades escénicas. Una evidencia empírica. $D Y O, 25,80-87$.

D'Agostino, P. (2010). The digital stage. Opera production for cinema and other audiovisual formats, a conversation with. International Workshop on Opera and Video. Valencia: Universidad Politécnica de Valencia/ Institut Valencià de la Música.

De Diego, A. (2010). Nuevas estrategias comunicativas en ópera por la inclusión del "making of" en los DVDs. International Workshop on Opera y Video. Valencia: Universidad Politécnica de Valencia/ Institut Valencià de la Música. Recuperado de http://us.academia.edu/AntonioDeDiego/Talks/34447/_New_ Communication_Strategies_at_Opera_by_the_inclusion_of_Making_of_in DVDs

Esse, M. ${ }^{-}$(2010). Don't Look Now: Opera, Liveness, and the Televisual. Opera Quarterly, 26 (1), 81-95. doi:10.1093/oq/kbq014

Fawkes, R. (2000). Opera on Film. London: Gerald Duckworth \& Co.

Fernández Quijada, D. (2007). Industrias culturales en el entorno digital: una reformulación desde la praxis comunicativa. Zer, 12 (22), 119-140.

Ferrés, J. (1997). Televisión, consumo y emociones. En J.L. Aguaded (Ed.), La otra mirada de la tele: pistas para un consumo inteligente de la televisión (pp. 47-56). Sevilla: Junta de Andalucía.

Fontcuberta, M. (2003). Medios de comunicación y gestión del conocimiento. Revista Iberoamericana de Educación, 32. Recuperado de http://www.rieoei.org/rie32a05. $\mathrm{htm}$

Fontcuberta, M. (2005). Medios de Comunicación y sociedad compleja: un desafío para la educación del siglo XXI. Pensamiento Educativo, 37, 142-162.

Fuenes Goyanes, E. (2010). El proyecto Opera-Oberta en la educación superior universitaria. El programa pedagógico en la URV, 2001-2008. Revista de Ciències de l'Educació, 35, 43-54. 
Föhl, P. \& Lutz, M. (2010). Publikumsforschung in öffentlichen Theatern und Opern. Nutzen, Bestandsaufnahme und Ausblick. En P. Glogner \& P. Föhl. Das Kulturpublikum (pp. 23-96). Wiesbaden: VS Verlag.

García Avilés, J. (2009). La comunicación ante la convergencia digital. Signo y Pensamiento, 54, 102-113.

Gaudio, A. (2009). Productions for Young Audiences in Europe. Brussels: RESEO.

Gordillo, I. (2008). La Hibridación en las nuevas formas dramáticas y espectaculares del siglo XXI. Icono14, 10, 1-20.

Hasebrink, U., Mikos, L. \& Prommer, E. (2004). Mediennutzung in konvergierenden Medienumgebungen, Band I. Múnich: Reinhard Fischer Verlag.

Herrscher, R. (2009, noviembre 25). En directo: pantallas para el bel canto. La Vanguardia, A24, A25.

Heyer, P. (2008). Live from the Met: Digital Broadcast Cinema, Medium Theory, and Opera for the Masses. Canadian Journal of Communication, 33, 591-604.

Hickethier, K. (2010). Mediatisierung und Medialisierung der kultur. En M. Hartmann \& A. Hepp (Eds.), Die Medietisierung der Allgswelt (pp. 85-96). Wiesbaden: VS Verlag.

Ishaghpour, Y. (1995). Opéra et théâtre dans le cinéma d'aujourd'hui. París: La Différence.

Jenkins, H. (2008). Convergence culture: La cultura de la convergencia de los medios de comunicación. Barcelona: Paidós.

Krotz, F. (2007). Mediatisierung. Fallstudien zum Wandel von KommunikationAuthor. Wiesbaden: VS Verlag.

Lacasa, I. \& Villanueva, I. (2011). La digitalización audiovisual de la ópera. Nuevos medios, nuevos usos, nuevos públicos. Telos, 88, 65-74.

Laenen, A. (2003). Why Opera Education?: a RESEO research report. Antwerp: RESEO.

Lipovetsky, G. \& Serroy, J. (2009). La pantalla global. Cultura mediática y cine en la era hipermoderna. Barcelona: Anagrama.

Lundby, K. (2009). Mediatization: concept, changes, consequences. New York: Peter Lang Publishing.

Marcos Ramos, M. (2010). Alfabetización mediática. La educación en los medios de comunicación: cine formativo y televisión educativa. Revista Electrónica Teoría de la Educación: Educación y Cultura en la Socidedad de la Información, 11 (2), 303-321.

Martel, F. (2011). Cultura “mainstream”: cómo nacen los fenómenos de masas. Madrid: Taurus.

Martín Barbero, J. (2002). Jóvenes: comunicación e identidad. Pensar Iberoamérica, 0. Recuperado de http://www.oei.es/pensariberoamerica/ric00a03.htm

Mclean, F. (1994). Services marketing: the case of museums. The Service Industries Journal, 14 (2), 190-203. doi:10.1080/02642069400000022

Medrano Samaniego, C. \& Cortés Pascual, A. (2007). The Teaching and Learning of Values trough Television. Review of Education, 53, 5-21. 
Miège, B. (2006). La concentración en las industrias culturales y mediáticas (ICM) y los cambios en los contenidos. Cuadernos de Información y Comunicación, 11, $155-166$.

Mitry, J. (1978). Estética y psicología del cine. Vol.1, Las estructuras. Madrid: Siglo XXI.

Mordden, E. (1985). El espléndido arte de la Ópera. Buenos Aires: Javier Vergara Editor.

Morris, C. (2010). Digital Diva: Opera on video. Opera Quarterly, 26 (1), 96-119. doi: $10.1093 /$ oq/kbq002

Nöel, A. (2010). Opera Houses and Their Cooperation with Higher Education. Brussels: RESEO. Recuperado de http://www.reseo.org/site/index.php?oldpg=r ess\&lg=en\&pg=research1

O'Shaughnessy, L. (2006). New Technology in Opera Education: Uses and Aspirations Findings. RESEO. Recuperado de http://www.reseo.org/site/index.php?oldpg=r ess\&lg=en\&pg=research1

Parker, R. (1998). Historia ilustrada de la ópera. Barcelona: Paidós Ibérica.

Pereira Domínguez, C. \& Urpí Guercia, C. (2005). Cine y Juventud: una propuesta educativa integral. Revista de estudios de juventud, 68, 73-89.

Perez Tornero, J. M. (1994). El desafío educativo de la televisión. Para comprender y usar el medio. Barcelona: Paidós Papeles de Comunicación.

Radigales, J. (1999). L'Òpera. Música, teatre i espectacle. Barcelona: Enciclopedia Catalana.

Radigales, J. (2005). La ópera y el cine: afinidades electivas. En M. Olarte (Ed.), La música en los medios audiovisuales (pp. 59-84). Salamanca: Universitaria.

Radigales, J. \& Villanueva. I. (2011). Ópera y cine en España: la ópera como producto audiovisual. Retos para el siglo XXI. Comunicación presentada en el Simposio Internacional de Música y Cine. Salamanca.

Ramírez, J. (2011). Entrevista realizada por los autores al Young Delegate de Opera Europa. Barcelona.

Romea, C. (2001). Lectura a cinco bandas:-La lengua de las mariposas-. Comunicar, 17, 71-78.

Rössel, J. (2011). Cultural Capital and the Variety of Modes of Cultural Consumption in the Opera Audience. The Sociological Quarterly, 52, 83-103. doi:10.1111/ j.1533-8525.2010.01192.x

Roselli, J. (1998). La ópera como acontecimiento social. En R. Parker (Ed.), Historia ilustrada de la ópera (pp.450-482). Barcelona: Paidós Ibérica.

Sánchez, S. (2008, mayo). La ópera en pantalla grande. Entrevista a José Batlle. Opera Actual, 116, 36-37.

Scheff, J. \& Kotler, P. (1996). Crisis in the arts: the marketing response. California Management Review, 39 (1), 28-52.

Schroeder, D. (2002). Cinema's Illusions, Opera's Allure. New York: The Continuum International Publishing Froup.

Senici, E. (2010). Porn Style? Space and Time in Live Opera Videos. Opera Quarterly, 26 (1), 63-80. doi:10.1093/oq/kbq010 
Silverstone, R. (1994). Television and Everyday Life. New York: Routledge.

Smith, J. (1993). Opera as an Interdisciplinary Art. Music Educators Journal, 79, 2161. doi:10.2307/3398544

Solé Blanch, J. (2005). Antropología de la educación y pedagogía de la juventud. Procesos de enculturación. Barcelona: Universitat Rovira i Virgili. Recuperado de http://www.tesisenred.net/handle/10803/8914;jsessionid=0835371F5ADBDE 4D80E35256EECA5907.tdx2

Tambling, J. (1987). Opera, Ideology and Film. New York: St. Martin's Press.

Tomlinson, G. (2001). Canto metafísico: un ensayo sobre la ópera. Barcelona: Idea Books.

Yorke, D.A. \& Jones, R.R. (1984). Marketing and Museums. European Journal of Marketing, 18 (2), 9. doi:10.1108/EUM0000000004773 